\title{
Hemograma e hemogasometria de eqüinos submetidos à obstrução experimental de jejuno
}

\author{
[Hemogram and blood gas analysis of equines submitted to jejuni experimental obstruction] \\ N.S. Costa ${ }^{1}$, G. Ribeiro ${ }^{1}$, R.G.S. Dória ${ }^{1}$, P.A. Canola ${ }^{1}$, P.C. Silva ${ }^{2}$, R.L.N. Jorge ${ }^{2}$, J.J. Fagliari ${ }^{3 *}$ \\ ${ }^{1}$ Aluno de pós-graduação - UNESP - Jaboticabal, SP \\ ${ }^{2}$ Laboratório de Apoio à Pesquisa - DCCV-UNESP - Jaboticabal, SP \\ ${ }^{3}$ Faculdade de Ciências Agrárias e Veterinárias - UNESP \\ Via de Acesso Prof. Paulo Donato Castellane, s/n \\ 14884-900 - Jaboticabal-SP
}

\begin{abstract}
RESUMO
Foram utilizados oito eqüinos distribuídos em dois grupos, submetidos ou não à obstrução experimental do jejuno mediante a colocação de um balão intraluminal. Os animais do grupo 1 foram submetidos à enterotomia com colocação do balão sem distensão suficiente para provocar isquemia e os do grupo 2 à isquemia por obstrução do jejuno durante quatro horas. Para determinação do hemograma e da hemogasometria foram obtidas amostras de sangue venoso em quatro momentos: uma hora antes do procedimento cirúrgico (M1), ao final da obstrução/isquemia (M2) e uma hora (M3) e 18 horas (M4) após o início da reperfusão/desobstrução. Não houve diferença entre grupos nas contagens de hemácias, leucócitos totais, neutrófilos bastonetes, neutrófilos segmentados, linfócitos e monócitos, bem como no teor de hemoglobina. Houve diferença no volume globular em M2 e na contagem de eosinófilos em M3. $\mathrm{Na}$ hemogasometria, em ambos os grupos, registrou-se diminuição dos teores de sódio, potássio, cálcio ionizado e cloro, da pressão parcial de oxigênio e da saturação de oxiemoglobina. Os resultados indicam que o hemograma, isoladamente, não fornece informações conclusivas, enquanto a hemogasometria possibilita determinar, precocemente, o volume de reposição hidroeletrolítica a ser administrado em eqüino com abdômen agudo decorrente de obstrução de jejuno, auxiliando no tratamento e no melhor prognóstico da afecção intestinal.
\end{abstract}

Palavras-chave: eqüino, hemograma, hemogasometria, obstrução de jejuno

\begin{abstract}
Eight equines were distributed into two different groups. Those groups were submitted or not to an experimental jejunal obstruction. Animals from group 1 were submitted to an enterotomy with placement of an intraluminal balloon, without enough distention to cause ischemia; animals from group 2 were submitted to ischemia by jejunal obstruction during four hours. In order to determine the hemogram and blood gas analysis, blood samples from peripheral vein were obtained at four moments: one hour before the surgical procedure (M1); at the end of obstruction/ischemia (M2); one hour (M3) and 18 hours (M4) after the beginning of reperfusion/deobstruction. There was no difference among the groups related to the counting of erythrocytes, hemoglobin, total white cells, neutrophils (band), neutrophils (segmented), lymphocytes, and monocytes. There was difference in the globular volume in M2 and in the eosinophils counting in M3. In both groups, the blood gas analysis identified diminished of sodium, potassium, ionized calcium, and chloride amounts, and partial pressure of oxygen and oxihemoglobin saturation. The results indicate that the hemogram alone, do not give conclusive information while the blood gas analysis allows an early determination of the volume of hydroelectrolytic replacement to be given to one equine with acute abdomen due to jejunal obstruction, providing additional support to the treatment and a better prognosis to an intestinal affection.
\end{abstract}

Keywords: horse, hemogram, blood, gas, jejuni obstruction

Recebido em 2 de abril de 2008

Aceito em 27 de novembro de 2008

*Autor para correspondência (corresponding author)

E-mail: fagliari@fcav.unesp.br 


\section{INTRODUÇÃO}

A síndrome abdômen agudo é uma das principais doenças dos eqüinos, colocando em risco a vida do paciente quando não se institui rapidamente um tratamento adequado. As afecções decorrentes de isquemia e de reperfusão constituem cerca de $50 \%$ dos casos desse distúrbio, com taxas de óbito de 66 a $75 \%$ (Susko, 1993). Com o incremento de informações sobre lesões isquêmicas difundiu-se o conceito de que a reperfusão em tecidos isquêmicos, apesar de essencial para prevenir a morte celular por anoxia, causa as chamadas lesões de reperfusão, que, por sua vez, agravam as lesões preexistentes (Ducharme et al., 1983). A dinâmica do mecanismo de morte celular no jejuno submetido a estrangulamento vascular em períodos de isquemia e congestão seguida de reperfusão foi demonstrada por Abreu et al. (2007). Esses autores verificaram que a apoptose ocorre em taxas fisiológicas, sendo também desencadeada pelo retorno circulatório.

Os resultados do hemograma, isoladamente, são inespecíficos, mas sua combinação com outros exames laboratoriais auxilia na caracterização da natureza do distúrbio e na elaboração do prognóstico, considerando o estado de hidratação, o risco cirúrgico, o tipo e a duração do processo inflamatório (Fagliari e Silva, 2002).

A hemogasometria é o método mais adequado para detecção de alterações do equilíbrio ácidobase dos fluidos orgânicos (Luna, 1994). Os desequilíbrios hidroeletrolíticos e ácido-base podem ser agravantes em eqüinos com doença gastrintestinal grave (Marques, 1990). No animal sadio, o volume e a composição dos fluidos orgânicos são mantidos em estreita faixa de variação. Nas enfermidades do trato digestório, os mecanismos de controle se alteram, sobrevindo desequilíbrios que incluem perda de água e de eletrólitos e concomitante distúrbio ácido-base. A correção dos distúrbios hidroeletrolíticos e ácido-base deve ser imediata, precedendo até mesmo a definição do diagnóstico e a decisão sobre a terapia específica a ser administrada (Ribeiro Filho, 2007).

O objetivo do estudo foi verificar possíveis alterações no hemograma e na hemogasometria de eqüinos submetidos à obstrução experimental do jejuno, a fim de se obter informações para o diagnóstico precoce da desvitalização do jejuno, o tratamento e o prognóstico da afecção intestinal.

\section{MATERIAL E MÉTODOS}

Foram utilizados oito eqüinos adultos, clinicamente hígidos, machos e fêmeas, sem raça definida, com cinco a 10 anos de idade e peso médio de $300 \mathrm{~kg}$. Os animais foram desverminados e mantidos, durante 10 dias, em piquete coletivo onde foi fornecida dieta diária composta de $5 \mathrm{~kg}$ de feno, por animal. Após esse período, foram distribuídos aleatoriamente em dois grupos de quatro animais. Os eqüinos do grupo $1(\mathrm{G} 1)$ foram submetidos à instrumentação cirúrgica semelhante aos do grupo 2, inclusive quanto à colocação do balão, porém sem distendê-lo a ponto de ocasionar obstrução do jejuno; os do grupo $2(\mathrm{G} 2)$ foram submetidos à instrumentação cirúrgica com colocação e distensão do balão, induzindo a obstrução intestinal e conseqüente isquemia do jejuno durante quatro horas.

Os animais, submetidos a jejum alimentar prévio de 18 horas, foram contidos em brete apropriado, sedados com cloridrato de acepromazina $^{1}$ $(0,025 \mathrm{mg} / \mathrm{kg}$, via intravenosa), cloridrato de xilazina $10 \%{ }^{2} \quad(0,5 \mathrm{mg} / \mathrm{kg}$, via intravenosa), meperidina $^{3}(2 \mathrm{mg} / \mathrm{kg}$, via intramuscular $) \mathrm{e}$ morfina $^{3}(0,1 \mathrm{mg} / \mathrm{kg}$ via epidural). A seguir, foi realizada tricotomia ampla da fossa paracostal direita seguida de rigorosa antissepsia com polivinipirrolidona iodo degermante por cinco minutos e remoção com álcool iodado. Logo após, procedeu-se à anestesia local por meio da técnica de "L" invertido, utilizando uma associação (1:1) de lidocaína $2 \%$ sem vasoconstritor $^{3}$ e bupivacaína $0,5 \%{ }^{3}$, conforme recomendação de Faleiros (2003).

Mediante laparotomia pelo flanco o jejuno foi exposto e submetido à obstrução intramural da parede intestinal com a implantação, no lúmen do jejuno, de um balão de látex por meio de enterotomia. A pressão no interior do balão foi registrada e monitorada por meio de um equipo comunicando o balão ao esfignomanômetro aneróide. Nos animais do grupo 2, o balão foi

\footnotetext{
${ }^{1}$ Univet S.A. Ind. Veterinária - São Paulo, Brasil.

${ }^{2}$ Fort Dodge - Campinas, Brasil.

${ }^{3}$ Cristália Hospitalar - Itapira, Brasil.
} 
inflado com auxílio de uma pêra insufladora até atingir a pressão de $18 \mathrm{cmH}_{2} \mathrm{O}$, conforme descrição de Dabarainer et al. (2001). As enterorrafias foram realizadas por sutura simples contínua e invaginante padrão Cushing, com fio de poliglactilona 91000 , deixando sair o equipo por entre as bordas da ferida.

Para a realização do hemograma e da hemogasometria foram obtidas amostras de sangue venoso em frascos que continham ácido etilenodiamino tetracético dissódico por meio de venopunção jugular em quatro momentos: uma hora antes do procedimento cirúrgico (M1); ao final da isquemia/obstrução de quatro horas (M2); uma hora após a desobstrução, ou seja, após o inicio da reperfusão (M3) e 18 horas após a desobstrução (M4).

As contagens de hemácias e de leucócitos e o teor de hemoglobina foram obtidos em aparelho automático $^{4}$. O volume globular foi calculado com o uso de microtubos de $50 \mu 1$ submetidos à centrifugação a $1520 \mathrm{G}$ durante cinco minutos. Para o cálculo dos índices hematimétricos volume corpuscular médio (VCM) e concentração de hemoglobina corpuscular média (CHCM) foram utilizadas fórmulas matemáticas elaboradas para tal fim (Thrall, 2007). Para a contagem diferencial de leucócitos foram preparados esfregaços sangüíneos $\operatorname{corados}^{5}$, contando-se 200 células, em microscopia óptica. Para a hemogasometria foi utilizado o equipamento Roche Omni C, mensurando-se os valores de sódio $\left(\mathrm{Na}^{+}\right)$, potássio $\left(\mathrm{K}^{+}\right)$, cálcio ionizado $\left(\mathrm{iCa}^{++}\right)$, cloro $\left(\mathrm{Cl}^{-}\right), \mathrm{pH}$, pressão parcial de oxigênio $\left(\mathrm{p}_{\mathrm{v}} \mathrm{O}_{2}\right)$, pressão parcial de gás carbônico $\left(\mathrm{p}_{\mathrm{v}} \mathrm{CO}_{2}\right)$, teor de bicarbonato $\left(\mathrm{HCO}_{3}^{-}\right)$, saturação de oxiemoglobina $\left(\mathrm{sO}_{2}\right)$ e excesso de base (EB). Também foi calculado o anion gap sangüíneo.

Realizou-se análise de variância de medidas repetidas com um fator $\mathrm{A}$ de dois níveis, ou seja, dois grupos experimentais, cada qual com quatro eqüinos, e um fator $\mathrm{B}$, representado pelos momentos das coletas de sangue, conforme recomendação de Zar (1999).

\section{RESULTADOS E DISCUSSÃO}

Os valores do eritrograma e do leucograma são apresentados nas Tab. 1 e 2, respectivamente.

No eritrograma, observaram-se valores mais elevados 18 horas após o início da reperfusão, ou seja, 18 horas após a desobstrução experimental (M4), sugerindo hemoconcentração. É possível, também, que as diminuições do teor de hemoglobina e do volume globular em M2 e M3 sejam decorrentes da ação da acepromazina. Este fato foi também relatado por Puotunen-Reinert e Huskamp (1986), Susko (1993) e Fagliari e Silva (2002). Jain (1993) descreveu que anestésicos como as fenotiazinas causam depleção do volume globular, podendo também ser observado decréscimo concomitante na concentração da proteína plasmática.

No leucograma, não ocorreram alterações significativas nos valores médios dos leucócitos totais, neutrófilos bastonetes e monócitos. No grupo II, ocorreu, entre os momentos, aumento nos valores médios de neutrófilos segmentados a partir de M2, situação que perdurou ao longo do experimento. Quanto aos linfócitos, também no grupo II, notou-se decréscimo significativo a partir de M2. Segundo Jain (1993) e Thrall (2007), a linfopenia observada é característica de afecções inflamatórias. Os valores de eosinófilos exibiram elevação em M3 e posterior diminuição em M4. Fagliari e Silva (2002) também constataram elevação de eosinófilos, em diferentes momentos, em animais submetidos à correção cirúrgica do quadro de abdômen agudo, no entanto, sem a diminuição subseqüente registrada neste ensaio. A única diferença entre grupos foi relativa ao aumento na contagem de eosinófilos em M3 nos animais do grupo II.

Segundo White II (1990), a hemogasometria venosa é adequada para determinar o equilíbrio ácido-base, auxilia na avaliação da gravidade do choque e pode indicar a presença de afecção no intestino delgado. Os valores relativos às análises de íons sódio, potássio, cálcio ionizado, cloro, bicarbonato, excesso de base, anion gap, $\mathrm{pH}$, pressão parcial de oxigênio, pressão parcial de gás carbônico e saturação de oxiemoglobina são apresentados na Tab. 3.

\footnotetext{
${ }^{4}$ ABX Diagnostics - Vet. - França.

${ }^{5}$ Laborclin Ltda. - Pinhais, Brasil.
} 
Tabela 1. Média e desvio-padrão da contagem de hemácias (He), concentração de hemoglobina ( $\mathrm{Hb})$, volume globular (VG), volume globular médio (VGM) e concentração de hemoglobina corpuscular média (CHCM) de eqüinos do grupo-controle (G1) e de eqüinos submetidos à isquemia de jejuno durante quatro horas (G2), uma hora antes do procedimento cirúrgico e aplicação de medicamento (M1), ao final da isquemia (M2), uma hora após a reperfusão (M3) e 18 horas após a reperfusão (M4)

\begin{tabular}{|c|c|c|c|c|}
\hline \multirow{2}{*}{ Constituinte } & \multicolumn{4}{|c|}{ Momentos } \\
\hline & M1 & M2 & M3 & M4 \\
\hline \multicolumn{5}{|l|}{$\mathrm{He} / \mu \mathrm{l}\left(\mathrm{x} 10^{6}\right)$} \\
\hline G1 & $6,48 \pm 1,47 \mathrm{Aa}$ & $6,10 \pm 1,68 \mathrm{Aa}$ & $5,77 \pm 1,68 \mathrm{Aa}$ & $7,60 \pm 1,09 \mathrm{Aa}$ \\
\hline G2 & $6,92 \pm 1,55 \mathrm{Aa}$ & $7,09 \pm 1,17 \mathrm{Aa}$ & $6,84 \pm 1,28 \mathrm{Aa}$ & $7,22 \pm 0,81 \mathrm{Aa}$ \\
\hline \multicolumn{5}{|l|}{$\mathrm{Hb}(\mathrm{g} / \mathrm{dl})$} \\
\hline G1 & $9,37 \pm 2,11 \mathrm{Aa}$ & $8,27 \pm 2,06 \mathrm{Aab}$ & $8,0 \pm 1,82 \mathrm{Aab}$ & $10,9 \pm 2,15 \mathrm{Aac}$ \\
\hline G2 & $8,97 \pm 0,34 \mathrm{Aa}$ & $9,32 \pm 2,16 \mathrm{Aa}$ & $9,22 \pm 2,12 \mathrm{Aa}$ & $11,3 \pm 1,60 \mathrm{Aa}$ \\
\hline \multicolumn{5}{|l|}{ VG (\%) } \\
\hline G1 & $27,0 \pm 5,65 \mathrm{Aa}$ & $23,5 \pm 4,12 \mathrm{Ab}$ & $23,0 \pm 3,55 \mathrm{Ab}$ & $32,2 \pm 5,67 \mathrm{Ac}$ \\
\hline G2 & $31,0 \pm 4,76 \mathrm{Aa}$ & $32,5 \pm 4,65 \mathrm{Ba}$ & $30,2 \pm 4,11 \mathrm{Ba}$ & $33,2 \pm 4,64 \mathrm{Aa}$ \\
\hline \multicolumn{5}{|l|}{ VGM (fL) } \\
\hline G1 & $42,9 \pm 10,4 \mathrm{Aa}$ & $40,4 \pm 11,8 \mathrm{Aa}$ & $42,2 \pm 11,9 \mathrm{Aa}$ & $42,7 \pm 2,75 \mathrm{Aa}$ \\
\hline G2 & $45,2 \pm 3,19 \mathrm{Aa}$ & $45,9 \pm 2,51 \mathrm{Aa}$ & $44,6 \pm 4,69 \mathrm{Aa}$ & $45,9 \pm 3,05 \mathrm{Aa}$ \\
\hline \multicolumn{5}{|l|}{ CHCM (\%) } \\
\hline G1 & $35,1 \pm 7,76 \mathrm{Aa}$ & $35,8 \pm 11,3 \mathrm{Aa}$ & $35,5 \pm 9,83 \mathrm{Aa}$ & $33,8 \pm 1,57 \mathrm{Aa}$ \\
\hline G2 & $29,3 \pm 3,90 \mathrm{Aa}$ & $28,7 \pm 5,01 \mathrm{Aa}$ & $30,4 \pm 4,73 \mathrm{Aa}$ & $34,0 \pm 1,29 \mathrm{Ab}$ \\
\hline
\end{tabular}

Letras maiúsculas distintas, nas linhas, indicam diferenças significativas entre os grupos $(\mathrm{P}<0,05)$.

Letras minúsculas distintas, nas colunas, indicam diferenças significativas entre os momentos $(\mathrm{P}<0,05)$.

Tabela 2. Média e desvio-padrão da contagem de leucócitos (Le), neutrófilos segmentados (NS), neutrófilos bastonetes (NB), linfócitos, monócitos e eosinófilos de eqüinos do grupo-controle (G1) e de eqüinos submetidos à isquemia de jejuno durante quatro horas (G2), uma hora antes do procedimento cirúrgico e aplicação de medicamento (M1), ao final da isquemia (M2), uma hora após a reperfusão (M3) e 18 horas após a reperfusão (M4)

\begin{tabular}{lcccc}
\hline \multirow{2}{*}{ Constituinte } & \multicolumn{4}{c}{ Momentos } \\
\cline { 2 - 5 } Le/ $\mu 1\left(\mathrm{x} 10^{3}\right)$ & & $\mathrm{M} 2$ & $\mathrm{M} 3$ & $\mathrm{M} 4$ \\
\hline G1 & $7,59 \pm 2,20 \mathrm{Aa}$ & $6,55 \pm 3,89 \mathrm{Aa}$ & $6,65 \pm 3,31 \mathrm{Aa}$ & $8,75 \pm 4,04 \mathrm{Aa}$ \\
G2 & $8,50 \pm 0,52 \mathrm{Aa}$ & $9,40 \pm 1,45 \mathrm{Aa}$ & $10,3 \pm 2,59 \mathrm{Aa}$ & $14,4 \pm 4,64 \mathrm{Aa}$ \\
NS/ $\mu 1$ & & & & \\
G1 & $64,2 \pm 17,1 \mathrm{Aa}$ & $66,75 \pm 12,0 \mathrm{Aa}$ & $73,7 \pm 5,67 \mathrm{Aa}$ & $60,0 \pm 18,9 \mathrm{Aa}$ \\
G2 & $62,2 \pm 7,71 \mathrm{Aa}$ & $76 \pm 10,0 \mathrm{Ab}$ & $79,5 \pm 4,04 \mathrm{Ab}$ & $81,5 \pm 5,06 \mathrm{Ab}$ \\
NB/ $/ \mu 1$ & $2,25 \pm 3,86 \mathrm{Aa}$ & $1,50 \pm 1,00 \mathrm{Aa}$ & $3,25 \pm 3,40 \mathrm{Aa}$ & $1,75 \pm 1,70 \mathrm{Aa}$ \\
G1 & $0 \pm 0 \mathrm{Aa}$ & $1,75 \pm 1,50 \mathrm{Aa}$ & $1,50 \pm 1,29 \mathrm{Aa}$ & $4,50 \pm 5,06 \mathrm{Aa}$ \\
G2 & & & & \\
Linfócitos$/ \mu 1$ & $30,5 \pm 18,0 \mathrm{Aa}$ & $30,5 \pm 11,3 \mathrm{Aa}$ & $21,5 \pm 6,45 \mathrm{Aa}$ & $36,5 \pm 18,6 \mathrm{Aa}$ \\
G1 & $33,5 \pm 7,14 \mathrm{Aa}$ & $21,0 \pm 9,30 \mathrm{Ab}$ & $15,0 \pm 4,54 \mathrm{Ab}$ & $13,2 \pm 4,71 \mathrm{Ab}$ \\
G2 & & & & \\
Monócitos$/ \mu 1$ & $2,25 \pm 1,70 \mathrm{Aa}$ & $1,00 \pm 0,81 \mathrm{Aa}$ & $1,00 \pm 0,81 \mathrm{Aa}$ & $1,00 \pm 0,50 \mathrm{Aa}$ \\
G1 & $1,25 \pm 0,50 \mathrm{Aa}$ & $0,25 \pm 0,50 \mathrm{Aa}$ & $1,00 \pm 1,15 \mathrm{Aa}$ & $0,75 \pm 0,50 \mathrm{Aa}$ \\
G2 & & & & \\
Eosinófilos $/ \mu 1$ & $0,75 \pm 0,95 \mathrm{Aa}$ & $0,25 \pm 0,5 \mathrm{Aa}$ & $0,25 \pm 0,5 \mathrm{Aa}$ & $0,75 \pm 1,50 \mathrm{Aa}$ \\
G1 & $3,00 \pm 1,82 \mathrm{Aa}$ & $1,00 \pm 0,81 \mathrm{Aa}$ & $3,00 \pm 1,41 \mathrm{Bab}$ & $0 \pm 0 \mathrm{Aac}$ \\
G2 & & &
\end{tabular}

Letras maiúsculas distintas, nas linhas, indicam diferenças significativas entre os grupos $(\mathrm{P}<0,05)$.

Letras minúsculas distintas, nas colunas, indicam diferenças significativas entre os momentos $(P<0,05)$. 
Tabela 3. Média e desvio-padrão dos valores de sódio $\left(\mathrm{Na}^{+}\right)$, potássio $\left(\mathrm{K}^{+}\right)$, cálcio ionizado $\left(\mathrm{iCa}^{++}\right)$, cloro $\left(\mathrm{Cl}^{-}\right)$, bicarbonato $\left(\mathrm{cHCO}_{3}^{-}\right)$, excesso de base (EB), anion gap $(\mathrm{AG}), \mathrm{pH}$, pressão parcial de oxigênio $\left(\mathrm{p}_{\mathrm{v}} \mathrm{O}_{2}\right)$, da pressão parcial de gás carbônico $\left(\mathrm{p}_{\mathrm{v}} \mathrm{CO}_{2}\right)$ e da saturação de oxiemoglobina $\left({ }_{\mathrm{s}} \mathrm{O}_{2}\right)$ de sangue total de eqüinos do grupo-controle (G1) e de eqüinos submetidos à isquemia de jejuno durante quatro horas (G2), uma hora antes do procedimento cirúrgico e aplicação de qualquer medicamento (M1), ao final da isquemia (M2), uma hora após a reperfusão (M3) e 18 horas após a reperfusão (M4)

\begin{tabular}{|c|c|c|c|c|}
\hline \multirow{2}{*}{ Constituinte } & \multicolumn{4}{|c|}{ Momento } \\
\hline & M1 & M2 & M3 & M4 \\
\hline \multicolumn{5}{|l|}{$\mathrm{Na}(\mathrm{mmol} / \mathrm{l})$} \\
\hline G1 & $134,8 \pm 4,10 \mathrm{Aa}$ & $132,3 \pm 2,79 \mathrm{Ab}$ & $132,8 \pm 2,49 \mathrm{Ab}$ & $131,8 \pm 4,50 \mathrm{Ab}$ \\
\hline $\mathrm{G} 2$ & $132,6 \pm 1,99 \mathrm{Aa}$ & $131,4 \pm 2,41 \mathrm{Aab}$ & $130,6 \pm 2,74 \mathrm{Aab}$ & $128,4 \pm 2,03 \mathrm{Ab}$ \\
\hline \multicolumn{5}{|l|}{$\mathrm{K}(\mathrm{mmol} / \mathrm{l})$} \\
\hline G1 & $4,19 \pm 0,48 \mathrm{Aa}$ & $3,51 \pm 0,52 \mathrm{Aa}$ & $3,45 \pm 0,57 \mathrm{Aa}$ & $3,46 \pm 0,50 \mathrm{Aa}$ \\
\hline G2 & $4,38 \pm 1,27 \mathrm{Aa}$ & $3,64 \pm 0,45 \mathrm{Aab}$ & $3,44 \pm 0,33 \mathrm{Aab}$ & $3,05 \pm 0,54 \mathrm{Ab}$ \\
\hline \multicolumn{5}{|l|}{$\mathrm{iCa}(\mathrm{mmol} / \mathrm{l})$} \\
\hline G1 & $1,449 \pm 0,19 \mathrm{Aa}$ & $1,342 \pm 0,13 \mathrm{Ab}$ & $1,250 \pm 0,178 \mathrm{Ab}$ & $1,344 \pm 0,160 \mathrm{Aab}$ \\
\hline G2 & $1,541 \pm 0,17 \mathrm{Aa}$ & $1,380 \pm 0,15 \mathrm{Ab}$ & $1,351 \pm 0,151 \mathrm{Abd}$ & $1,396 \pm 0,183 \mathrm{Ac}$ \\
\hline \multicolumn{5}{|l|}{$\mathrm{Cl}(\mathrm{mmol} / \mathrm{l})$} \\
\hline G1 & $97,5 \pm 0,97 \mathrm{Aa}$ & $94,9 \pm 2,04 \mathrm{Aa}$ & $92,7 \pm 2,77 \mathrm{Aa}$ & $94,1 \pm 5,03 \mathrm{Aa}$ \\
\hline G2 & $96,4 \pm 2,31 \mathrm{Aa}$ & $89,4 \pm 2,97 \mathrm{Bb}$ & $88,6 \pm 1,88 \mathrm{Bb}$ & $89,4 \pm 4,21 \mathrm{Ab}$ \\
\hline \multicolumn{5}{|l|}{$\mathrm{HCO}_{3}(\mathrm{mmol} / \mathrm{l})$} \\
\hline G1 & $28,3 \pm 1,77 \mathrm{Aa}$ & $29,3 \pm 2,80 \mathrm{Aab}$ & $31,8 \pm 1,99 \mathrm{Ab}$ & $26,6 \pm 2,24 \mathrm{Aac}$ \\
\hline G2 & $29,9 \pm 2,47 \mathrm{Aa}$ & $34,8 \pm 1,54 \mathrm{Bb}$ & $35,0 \pm 1,14 \mathrm{Ab}$ & $30,4 \pm 2,59 \mathrm{Aa}$ \\
\hline \multicolumn{5}{|l|}{$\mathrm{EB}(\mathrm{mmol} / \mathrm{l})$} \\
\hline G1 & $3,32 \pm 1,45 \mathrm{Aa}$ & $5,35 \pm 3,08 \mathrm{Aab}$ & $6,85 \pm 2,85 \mathrm{Ab}$ & $3,13 \pm 0,73 \mathrm{Aa}$ \\
\hline G2 & $4,20 \pm 2,16 \mathrm{Aa}$ & $8,37 \pm 1,16 \mathrm{Ab}$ & $9,50 \pm 1,02 \mathrm{Ab}$ & $5,67 \pm 2,43 \mathrm{Aa}$ \\
\hline \multicolumn{5}{|l|}{$\mathrm{AG}(\mathrm{mmol} / \mathrm{l})$} \\
\hline G1 & $13,1 \pm 2,75 \mathrm{Aa}$ & $12,9 \pm 1,30 \mathrm{Aa}$ & $12,4 \pm 1,20 \mathrm{Aab}$ & $14,5 \pm 1,70 \mathrm{Aac}$ \\
\hline $\mathrm{G} 2$ & $10,6 \pm 1,04 \mathrm{Aa}$ & $10,7 \pm 2,28 \mathrm{Aa}$ & $10,3 \pm 2,34 \mathrm{Aa}$ & $11,6 \pm 0,78 \mathrm{Ba}$ \\
\hline \multicolumn{5}{|l|}{$\mathrm{pH}$} \\
\hline G1 & $7,41 \pm 0,07 \mathrm{Aa}$ & $7,47 \pm 0,41 \mathrm{Aa}$ & $7,45 \pm 0,54 \mathrm{Aa}$ & $7,42 \pm 0,22 \mathrm{Aa}$ \\
\hline G2 & $7,40 \pm 0,15 \mathrm{Aa}$ & $7,40 \pm 0,06 \mathrm{Ba}$ & $7,45 \pm 0,28 \mathrm{Ab}$ & $7,44 \pm 0,15 \mathrm{Ab}$ \\
\hline \multicolumn{5}{|l|}{$\mathrm{p}_{\mathrm{v}} \mathrm{O}_{2}(\mathrm{mmHg})$} \\
\hline G1 & $39,07 \pm 3,06 \mathrm{Aa}$ & $34,37 \pm 1,74 \mathrm{Aa}$ & $30,95 \pm 2,26 \mathrm{Aa}$ & $37,82 \pm 5,24 \mathrm{Aa}$ \\
\hline G2 & $40,82 \pm 5,70 \mathrm{Aa}$ & $28,65 \pm 1,98 \mathrm{Bb}$ & $29,97 \pm 5,66 \mathrm{Ab}$ & $36,5 \pm 5,92 \mathrm{Aab}$ \\
\hline \multicolumn{5}{|l|}{$\mathrm{p}_{\mathrm{v}} \mathrm{CO}_{2}(\mathrm{mmHg})$} \\
\hline G1 & $46 \pm 2,27 \mathrm{Aa}$ & $40,7 \pm 2,94 \mathrm{Aa}$ & $46,6 \pm 4,44 \mathrm{Aa}$ & $42,1 \pm 3,95 \mathrm{Aa}$ \\
\hline G2 & $48,37 \pm 2,38 \mathrm{Aa}$ & $55,32 \pm 2,78 \mathrm{Bb}$ & $51,07 \pm 3,48 \mathrm{Aabc}$ & $45,07 \pm 3,55 \mathrm{Aad}$ \\
\hline \multicolumn{5}{|l|}{$\mathrm{sO}_{2}(\%)$} \\
\hline G1 & $72,77 \pm 5,14 \mathrm{Aa}$ & $70,97 \pm 9,21 \mathrm{Aa}$ & $64,2 \pm 11,97 \mathrm{Aa}$ & $70,55 \pm 6,69 \mathrm{Aa}$ \\
\hline G2 & $77,65 \pm 5,04 \mathrm{Aa}$ & $56,7 \pm 7,57 \mathrm{Bb}$ & $62,3 \pm 15,32 \mathrm{Aab}$ & $72,05 \pm 8,07 \mathrm{Aab}$ \\
\hline
\end{tabular}

Letras maiúsculas distintas, nas linhas, indicam diferenças significativas entre os grupos $(\mathrm{P}<0,05)$.

Letras minúsculas distintas, nas colunas, indicam diferenças significativas entre os momentos $(P<0,05)$.

Na hemogasometria de amostra de sangue total, observaram-se, em ambos os grupos, valores progressivamente diminuídos de sódio e potássio durante o período de obstrução (M2), uma hora após o início da reperfusão (M3) e 18 horas após a desobstrução (M4). Bordin et al. (2007), em trabalho com eqüinos hipovolêmicos, não observaram diminuição dos teores de sódio, potássio e cloro, o que remete a gravidade do quadro de obstrução de jejuno. Para Johnson
(1995), a hiponatremia persistente ocorre apenas quando há grave comprometimento da função renal, o que pode ter influenciado a magnitude dos valores observados, considerando que quase todo sódio filtrado é absorvido no túbulo renal proximal. Os sinais clínicos relacionados com hiponatremia grave são manifestações neurológicas associadas ao edema cerebral, inclusive letargia, tremores e convulsões, sinais estes evidenciados em alguns animais do estudo. 
A hiponatremia, segundo Haskins (1988), sugere excesso relativo de água, o que clinicamente pode induzir à fraqueza. Quanto aos sintomas de déficit de potássio, Seahorn e Seahorn (2003) também descrevem arritmias cardíacas, disfunção miocárdica e íleo paralítico. De acordo com Nappert e Johnson (2001), a hipocalemia é um achado freqüente em eqüinos com cólica, e para Fettman (2004), pode ser decorrente de fatores como alcalose, alto teor plasmático de insulina ou depleção do potássio corpóreo, informação que também foi citada por Seahorn e Seahorn (2003). Sabe-se que alguns hormônios, inclusive a insulina e as catecolaminas, podem induzir a perda de potássio, do mesmo modo que a excitação e a ativação da medular da adrenal podem resultar em hipocalemia. Haskins (1988) teoriza que a hipocalemia é bastante comum em afecções intestinais agudas.

Hipocalcemia e hipocloremia também foram achados comuns nos eqüinos dos dois grupos, ao longo do período experimental, com menores valores observados uma hora após a desobstrução, ou seja, no início da reperfusão. Estes resultados assemelham-se aos encontrados por Garcia-Lopez et al. (2001) em cães e seres humanos com doenças terminais. Trim (1990) citou que a hipocalcemia está relacionada à hipotensão e ao prejuízo à função cardiovascular. Múltiplos fatores, incluindo anorexia, endotoxemia e ressecção intestinal, podem estar associados à baixa concentração de cálcio em cães (Dart et al., 1992), condições estas comuns em eqüinos com abdômen agudo. Os autores citaram, ainda, que as endotoxinas ou seus subprodutos podem causar hipocalcemia durante a sepse, pelo prejuízo à mobilização desse cátion. Segundo Garcia-Lopez et al. (2001), a determinação do cálcio pode ser útil na avaliação da gravidade da lesão. As alterações observadas na mensuração do cloro acompanharam os desequilíbrios do sódio, corroborando as afirmações de Johnson (1995). Em relação à dinâmica do cloro, este é secretado ativamente no estômago e absorvido na porção final do intestino delgado, podendo ocorrer hipocloremia quando há grande refluxo gástrico, após lavagens gástricas repetidas e em distúrbios que comprometem sua absorção (Seahorn e Seahorn, 2003). White II (1990) relata que a hipocloremia pode estar associada ao seqüestro gastrintestinal ou ao refluxo gástrico, como acontece nas obstruções proximais do intestino delgado.
As concentrações de bicarbonato e cloro têm, em geral, relação inversa, ou seja, na alcalose metabólica há aumento do teor de bicarbonato e conseqüente diminuição da cloremia (Johnson, 1995), pois ocorrem alterações compensatórias de bicarbonato para manter a eletroneutralidade (Luna, 1994; Nappert e Johnson, 2001). Tal achado foi verificado em animais de ambos os grupos com maiores valores registrados nos animais do grupo 2 ao final da obstrução (M2) e uma hora após a reperfusão (M3). Segundo Carlson (1989), nos distúrbios metabólicos, como acontece no abdômen agudo em eqüino, a alteração inicial notada ocorre na concentração de bicarbonato. Sabe-se que o bicarbonato atua na neutralização dos íons $\mathrm{H}^{+}$, ingeridos ou produzidos constantemente pelo metabolismo celular. Quando o hidrogênio sangüíneo diminui o $\mathrm{pH}$ aumenta e o animal desenvolve alcalemia, o que confirma os achados de Svendsen et al. (1979), que verificaram alcalose metabólica em eqüinos com cólica.

Quanto ao excesso de base (EB), esta variável indica desvio do valor normal do teor de $\mathrm{HCO}_{3}{ }^{-}$ (Carlson, 1989). Não ocorreram alterações significativas nos valores médios de EB entre os grupos. No entanto, em M2 e M3 de ambos os grupos notou-se aumento significativo. Acreditase que tal elevação decorreu da somatória das alterações clínico-patológicas promovidas pela colocação do balão intraluminal no jejuno que, segundo White II (1990), pode implicar em seqüestro de íons hidrogênios no estômago.

$\mathrm{O}$ anion gap é definido como a diferença $\left(\mathrm{Na}^{+}+\right.$ $\left.\mathrm{K}^{+}\right)$- $\left(\mathrm{CL}^{-}+\mathrm{HCO}_{3}^{-}\right)$, de acordo com DiBartola (2001). Os dados obtidos a partir desta equação não mostraram diferenças significativas entre os grupos, tampouco entre momentos, com exceção daquele observado 18 horas após o inicio da reperfusão (M4) nos animais do grupo-controle. Tal fato pode ser atribuído à diminuição de $\mathrm{HCO}_{3}{ }^{-}$, aumento do teor de cloro e normalização do $\mathrm{pH}$, perfil que se assemelha ao relatado por Susko (1993), que encontrou relação inversa entre $\mathrm{HCO}_{3}{ }^{-}$e anion gap.

$\mathrm{O} \mathrm{pH}$ do sangue venoso, importante variável na determinação do estado do equilíbrio ácido-base em eqüinos, é considerado normal quando se situa entre 7,32 e 7,44 (Carlson, 1989). Nos animais do grupo 1, notou-se aumento significativo do $\mathrm{pH}$ na quarta hora após a 
colocação do balão (M2), quadro que não se manteve nos demais momentos. Nos do grupo 2, constatou-se diferença relevante uma hora após a desobstrução (M3), situação que perdurou até a $18^{\mathrm{a}}$ hora de reperfusão (M4), caracterizando o quadro de alcalose, o que confirma os achados de Svendsen et al. (1979) e Susko (1993).

Sabe-se que os pulmões, juntamente com os rins, são os principais órgãos envolvidos na regulação do sistema ácido-base e que a avaliação dos gases sangüíneos, $\mathrm{p}_{\mathrm{v}} \mathrm{O}_{2}$ e $\mathrm{p}_{\mathrm{v}} \mathrm{CO}_{2}$ determinam a intensidade de trocas gasosas no pulmão e a respiração celular, servindo de parâmetro comparativo no estudo do equilíbrio ácido-base (Carlson, 1989). Em relação às variáveis $\mathrm{p}_{\mathrm{v}} \mathrm{O}_{2} \mathrm{e}$ $\mathrm{p}_{\mathrm{v}} \mathrm{CO}_{2}$, verificaram-se diferenças significativas entre os grupos na quarta hora de isquemia (M2), sendo aferidos os menores valores de $\mathrm{p}_{\mathrm{v}} \mathrm{O}_{2}$ e os maiores valores de $\mathrm{p}_{\mathrm{v}} \mathrm{CO}_{2}$ nos animais submetidos à obstrução (G2). Nos animais do grupo 2, as alterações de $\mathrm{p}_{\mathrm{v}} \mathrm{O}_{2}$, estabelecidas em M2, M3 e M4, foram significativas quando comparadas aos valores do momento basal (M1). À semelhança da $\mathrm{p}_{\mathrm{v}} \mathrm{O}_{2}$ e da $\mathrm{p}_{\mathrm{v}} \mathrm{CO}_{2}$, a saturação de oxihemoglobina $\left(\mathrm{S}_{2} \mathrm{O}_{2}\right)$ não apresentou alteração nos animais do grupo 1 , enquanto os animais do grupo 2 demonstraram os menores valores de ${ }_{\mathrm{S}} \mathrm{O}_{2}$ na quarta hora de obstrução (M2).

Diante dos resultados da hemogasometria, e tendo como valores de referência os descritos por Carlson (1989), pode-se considerar que os animais do grupo 1 apresentaram alcalose metabólica quatro horas após a colocação do balão (M2) e uma hora após a sua retirada (M3) enquanto os do grupo 2 apresentaram quadro prolongado de alcalose durante todo o período de reperfusão (M3) e (M4), sendo em M3 o registro de valor mais elevado de $\mathrm{pH}$. Os resultados encontrados assemelham-se aos de Susko (1993), que detectou alcalose metabólica após uma hora de obstrução do intestino delgado. Essas alterações confirmam as verificadas por White II (1990) que citou que eqüinos com obstrução no intestino delgado apresentam alcalose devido à retenção de ácidos no estômago, resultado também encontrado por Puotunen-Reinert e Huskamp (1986).

\section{CONCLUSÃO}

Pode-se considerar que os resultados do hemograma, isoladamente, não sugerem informações conclusivas a cerca do quadro de abdômen agudo induzido. Quanto à hemogasometria, considera-se que sua principal aplicação é determinar a quantidade de fluido e eletrólitos de reposição ao eqüino com abdômen agudo, auxiliando a direcionar os procedimentos de fluidoterapia e colaborando com o tratamento, bem como no prognóstico da afecção intestinal.

\section{AGRADECIMENTOS}

Os autores agradecem à FAPESP pelo auxílio financeiro concedido e à CAPES pela bolsa de doutorado concedida.

\section{REFERÊNCIAS BIBLIOGRÁFICAS}

ABREU, J.M.G.; ALVES, G.E.S.; VASCONCELOS, A.C.. Efeitos da isquemia e da reperfusão na ocorrência de apoptose no jejuno em eqüinos. Arq. Bras. Med. Vet. Zootec., v.59, p.35-40, 2007.

BORDIN, A.I.; OLIVEIRA, P.; FREITAS, C.F. et al. Efeitos da solução salina hipertônica a $7,5 \%$ em glicose a 5\% nas concentrações séricas de sódio, cloreto e potássio de eqüinos com hipovolemia induzida. Arq. Bras. Med. Vet. Zootec., v.59, p.621-626, 2007.

CARLSON, G.P. Fluid, electrolyte, and acidbase balance. In KANEKO, J.J. (Ed). Clinical biochemistry of domestic animals. 4.ed. San Diego: Academic, 1989. p.543-575.

DABARAINER, R.M.; WHITE II, N.A.; DONALDSON, L.L. Effects of intraluminal distention and decompression on microvascular permeability and hemodynamics of the equine jejunum. Am. J. Vet. Res., v.62, p.225-236, 2001.

DiBARTOLA, S.P. Interpretation of metabolic acid base disturbances using the routine serum biochemical profile. J. Feline Med. Surg., v.3, p.189-191, 2001.

DART, A. J.; SNYDER, J.R.; SPIER, S.J. et al. Ionized calcium concentration in horses with surgically managed gastrointestinal disease: 147 cases (1988-1990). J. Am. Vet. Med. Assoc., v.201, p.1244-1248, 1992.

DUCHARME， N.G.; HACKETT, R.P.; DUCHARME, G.R. et al. Surgical treatment of colic: results in 181 horses. Vet. Surg., v.12, p.206-209, 1983. 
FAGLIARI , J.J.; SILVA, S.L. Hemograma e proteinograma plasmático de eqüinos hígidos e de eqüinos acometidos por abdômen agudo, antes e após laparotomia. Arq. Bras. Med. Vet. Zootec., v.54, p.559-567, 2002.

FALEIROS, R.R. Obstrução experimental do cólon menor eqüino: aspectos clínicos, patológicos e terapêuticos. 2003. 168f. Tese (Doutorado) - Faculdade de Ciências Agrárias e Veterinárias, Universidade Estadual Paulista, Jaboticabal.

FETTMAN, M.J. Fluid and electrolyte metabolism. In:_ Veterinary hematology and clinical chemistry. Philadelphia: Lippincott Williams \& Wilkis, 2004. p.329-355.

GARCIA-LOPEZ, J.M.; PROVOST, P.J.; RUSH, J.E. et al. Prevalence and prognostic importance of hypomagnesemia and hypocalcemia in horses that have colic surgery. Am. J. Vet. Res., v.62, p.7-12, 2001.

HASKINS, S.C. A simple fluid therapy planning guide. Semin. Vet. Med. Surg., v.3, p.227-236, 1988.

JAIN, N.C. Essentials of veterinary hematology. Philadelphia: Lea \& Febiger, 1993. p.222-295.

JOHNSON, P.J. Electrolyte and acid-base disturbances in the horse. Vet. Clin. N. Am.: Equine Pract., v.11, p.491-514, 1995.

LUNA, S.P.L. Interpretação de exames laboratoriais. Diagnóstico em cólica eqüina. In: FORUM DE GASTROENTEROLOGIA EQUINA, I., 1994, Curitiba. Anais... Curitiba CBCAV, 1994. p.38-48.

MARQUES, L.C. Equilíbrio hídrico-eletrolítico. Cólica Equina II: Diagnóstico e tratamento. In: CICLO DE PALESTRAS SOBRE CÓLICA EQUINA, II., 1990, Jaboticabal. Anais... Jaboticabal, 1990. (Bol. Tec. p.47-70).
NAPPERT, G.; JOHNSON, P. Determination of the acid-base status in 50 horses admitted with colic between December 1998 and May 1999. Can. Vet. J., v.42, p.703-707, 2001.

PUOTUNEN-REINERT, A.; HUSKAMP, B. Experimental duodenal obstruction in the horse. Vet. Surg., v.15, p.420-428, 1986.

RIBEIRO-FILHO, J.D.; ABREU, J.M.G.; ALVES, G.E.S. et al. Hemogasometria em eqüinos com compactação experimental do cólon maior tratados com sene, fluidoterapia enteral e parenteral. Cienc. Rural, v.37, p.755-761, 2007.

SEAHORN, J.L.; SEAHORN, T.L. Fluid therapy in horses with gastrointestinal disease. Vet. Clin. N. Am.: Equine Pract., v.9, p.577-604, 2003.

SUSKO, I. Modelo experimental de obstrução do terço médio do jejuno em eqüinos: avaliações clínicas, hematológicas, bioquímicas, hidroeletrolíticas, do equilibrio ácido-básico e anátomo-patológicas. 1993. 204f. Tese (Doutorado) - Faculdade de Medicina Veterinária e Zootecnia, Universidade Estadual Paulista, Botucatu.

SVENDSEN, C.K.; HJORTKJAER, R.K.; HESSELHOLT, M. et al. Colic in the horse: A clinical and clinical chemical study of 42 cases. Nord. Vet. Med., v.31, p.1-32, 1979.

THRALL, M.A. Hematologia e bioquímica clínica veterinária. São Paulo: Roca, 2007. p.329-354.

TRIM, C.M. Anesthesia for acute abdominal disease. In: WHITE II, N.A. (Ed). The equine acute abdomen. Philadelphia: Lea \& Febiger, 1990. p.189-207.

ZAR, J.H. Biostatistical analysis. New Jersey: Prentice Hall, 1999. 292p.

WHITE II, N.A. (Ed). The equine acute abdomen. Philadelphia: Lea \& Febiger, 1990. p.102-147. 